\title{
Application of Circular Polymerase Extension Cloning to Generate Infectious Clones of a Plant Virus
}

\author{
Dey K. Kishore \\ Department of Plant and Environmental Protection Sciences, \\ University of Hawaii at Manoa \\ 3190 Maile Way, Honolulu, HI, 96822, USA
}

W. B. Borth

Department of Plant and Environmental Protection Sciences,

University of Hawaii at Manoa

3190 Maile Way, Honolulu, HI, 96822, USA

\section{J. Melzer}

Department of Plant and Environmental Protection Sciences,

University of Hawaii at Manoa

3190 Maile Way, Honolulu, HI, 96822, USA

\author{
John S. Hu (Corresponding author) \\ Department of Plant and Environmental Protection Sciences, \\ University of Hawaii at Manoa \\ 3190 Maile Way, Honolulu, HI, 96822, USA \\ Tel: 1-808-956-7281; Fax: 1-808-956-2832 \\ E-mail: johnhu@hawaii.edu
}

Received: October 18, 2014 Accepted: December 10, 2014 Published: January 8, 2015

Doi: 10.5296/jab.v3n1.6455 URL: http://dx.doi.org/10.5296/jab.v3n1.6455 


\title{
II Macrothink
}

\begin{abstract}
Infectious clones of viruses allow elucidation of viral gene function in plants. This study presents an adaptation and expansion of the circular polymerase extension reaction that enables both cell-based and cell-free one-step assembly of a plant viral cDNA. To demonstrate its infectious nature, the generated clone was introduced into plants by Agrobacterium infiltration. This technique eliminates the cumbersome strategy of restriction enzyme digestion and ligation and instead relies on the sequence specificity of overlapping PCR fragments to assemble a complete functional infectious clone of a virus. This technique is rapid and potentially applicable for cloning virus genes which may be difficult to clone using conventional approaches due to toxicity problems that may be encountered when cloning in E. coli.
\end{abstract}

Keywords: PVX, Circular polymerase Extension cloning, Infectious clones, Viral toxicity, Plant viruses 


\section{Introduction}

Full-length cDNA infectious clones of viruses are important tools used to study the function of viral genomes at the molecular level. Despite advances made over the last few decades in the development of molecular techniques to clone large viral genomes, there is still no robust technique that is amenable to all types of viral genomes. The traditional approach to develop full-length infectious clones of RNA viruses utilizes unique restriction sites present in cDNA copies of viral genomes for the stepwise restriction-digestion and ligation of fragments generated by reverse transcription-PCR (RT-PCR). The availability of proof-reading Taq polymerases that can amplify long DNA fragments without introducing errors has reduced the number of fragments of viral genomes that must be assembled to construct full-length clones. The generation of full-length infectious clones using single PCR cycles (Youssef et al., 2011) has become routine for viruses with smaller-sized genomes. This has simplified the process, but a considerable amount of time and effort is still required to generate complete infectious clones. In instances where certain components of viral genomes are toxic to the $E$. coli strains used in cloning, these traditional approaches have serious constraints. For example, viruses in the genus Flavivirus, which includes medically important viruses such as West Nile virus, Japanese encephalitis virus, and Dengue virus, cell-based cloning has serious limitations due to bacterial toxicity of the cloned sequences (Lin et al., 2012). A number of plant viruses within the agriculturally important Potyviridae family contain genome components that have also been reported to be toxic to E. coli strains (López-Moya \& García, 2000). Different methods have been devised to overcome these toxicity problems (Satyanarayana et al., 2003) such as inserting introns into the clone (Johansen, 1996) (López-Moya \& García, 2000), cloning toxic regions as the final step in assembly (Jakab et al., 1997), or maintaining separate plasmids containing distinct genome fragments and ligating them in-vitro as the final step (Sumiyoshi et al., 1992). Another technique that uses the yeast recombination system to assemble full-length clones has been described (Youssef et al., 2011), but in all these cases, considerable time and effort must be invested to generate infectious clones of these viruses.

Circular Polymerase Extension Cloning (CPEC) has been shown to be very useful for assembling and cloning genes in addition to its use in high-throughput genomics and synthetic biology (Quan \& Tian, 2009; Quan \& Tian, 2011). A major appeal of CPEC is its simplicity. CPEC is based on extension of products from polymerase chain reactions (PCR), but unlike PCR, it does not amplify products exponentially but rather in a linear fashion using the overlapping regions of the DNA fragments as primers to extend the fragment. Quan and Tian (2009) first demonstrated such cloning by fusing an insert into a vector using Taq polymerase resulting in a complete circular double-stranded plasmid with nicks in each strand that were subsequently filled-in after introduction into the host.

CPEC has the potential to be a useful method for cloning the large genomes of some viruses which lack unique restriction sites thus making conventional cloning procedures problematic (Satyanarayana et al., 1999). CPEC also provides the flexibility of joining cDNAs from any location within a virus genome. In addition, the use of high-fidelity polymerases in both the RT-PCR and CPEC steps ensures that no unwanted mutations are incorporated into the assembled sequences. This ability to synthesize complex gene fragments by CPEC without resorting to traditional restriction digestion, ligation, and cloning steps was recently utilized 
to create an infectious cDNA clone of the Kunjin Strain of West Nile virus (KUNV) (Edmonds et al., 2013). The use of CPEC in that study was unique because the infectious clones that were generated recapitulated the genetic heterogeneity present in the original isolate (Edmonds et al., 2013). Infectious clones of Dengue virus have been constructed (Siridechadilok et al., 2013) using a similar approach known as Gibson Assembly ${ }^{\circledR}$ (Gibson et al., 2009). But in Gibson Assembly the joining of multiple fragments was carried out at an isothermal temperature and required a cocktail of enzymes that is not necessary in CPEC.

We have adapted the original CPEC technique and extended its use to clone a plant virus. We demonstrate that a full-length infectious clone of Potato virus $X$ (genus Potexvirus) could be generated in one week using CPEC. This is the first application of this approach to clone the complete genome of a plant virus, and demonstrates the potential for using this technique to clone other plant viruses.

\section{Methods}

The CPEC approach was adapted to generate infectious cDNAs of PVX cloned in Agrobacterium tumefaciens strain GV3101. The Ukraine strain of PVX (pGR106) that has been modified to include a multiple cloning site between the coat protein ( $\mathrm{CP}$ ) and $8 \mathrm{~K}$ protein (Lu et al., 2003) was used to infect Nicotiana benthamiana plants. To generate overlapping fragments of the PVX genome, total RNAs including complete PVX genomes were isolated from systemically infected leaves of pGR106-infected plants using an RNeasy ${ }^{\circledR}$ Plant Mini Kit (Qiagen, USA) and used as templates in RT-PCR. The pGR106 PVX plasmid template was used to generate vector fragments containing overlapping ends complementary to the ends of the virus genome (Figure 1). Primers were designed to have regions complementary to the first 108 bp of the 5'-terminus and 226 bp of the 3'-terminus of the PVX genome. Viral cDNAs for two or three overlapping fragments (referred to as ' $a$ ' and ' $b$ ' in Figure1), were synthesized using $10 \mu \mathrm{g}$ random hexamer primers (Promega, USA) and Superscript ${ }^{\circledR}$ reverse transcriptase (Invitrogen, USA), followed by amplification of cDNA fragments using Phusion ${ }^{\circledR}$ high-fidelity DNA polymerase (Thermo Scientific, USA) and primer-pairs designed to recognize individual fragments (Figure 1). The primers were designed to have similar annealing temperatures with the overlapping ends of two neighboring fragments and also with the other fragments. To prevent cross-joining of the RT-PCR fragments during the CPEC reaction, annealing temperatures were kept above $65^{\circ} \mathrm{C}$. The expected sizes of individual RT-PCR fragments were verified in $1.2 \%(\mathrm{w} / \mathrm{v})$ agarose gels stained with ethidium bromide. The RT-PCR products were then purified using a PCR purification kit (Qiagen) and quantified on an ND-1000 spectrophotometer (Nanodrop, USA). The CPEC reaction was performed using equimolar amounts $(\sim 200 \mathrm{ng})$ of all the RT-PCR products including the vector fragment. The total amount of DNA used was approximately $800 \mathrm{ng}$. CPEC reaction conditions were; denaturation for $45 \mathrm{sec}$ at $98^{\circ} \mathrm{C}$, followed by 15 cycles of denaturation for $15 \mathrm{sec}$ at $98^{\circ} \mathrm{C}$, annealing for $30 \mathrm{sec}$ at $65^{\circ} \mathrm{C}$, and extension for $7 \mathrm{~min}$ at $72^{\circ} \mathrm{C}$. Reaction volumes were $50 \mu 1$ and 10 pmol of each primer pair were used. $5 \mu 1$ of the CPEC reactions were analyzed in $1.2 \%$ agarose gels to confirm expected sizes, and $15 \mu \mathrm{l}$ was used to transform E. coli $\mathrm{DH} 5 \alpha$ cells that were then grown at $37^{\circ} \mathrm{C}$ on $\mathrm{LB}$ plates with kanamycin selection. Another $15 \mu \mathrm{l}$ aliquot of the PCR reactions was electroporated into Agrobacterium 
GV3101 electro-competent cells at 1500V using a 2510-Electroporator (Eppendorf, USA) and then grown in Luria Bertani (LB) media suplemented with $10 \mathrm{mM}$ MES, $10 \mu \mathrm{M}$ acetosyringone, and selection with $50 \mu \mathrm{g} / \mathrm{L}$ kanamycin and $25 \mu \mathrm{g} / \mathrm{L}$ rifampacin. These cultures were grown at $30^{\circ} \mathrm{C}$ overnight, harvested by centrifugation, and resuspended in infiltration media (10mM MES, $10 \mathrm{mM} \mathrm{MgCl}_{2}, 150 \mu \mathrm{M}$ acetosyringone) at an optical density $\left(\mathrm{OD}^{600}\right)$ of 1.0. The cultures were kept for 2-3 hours at room temperature before co-infiltrating the abxial surface of four-week old $N$. benthamiana leaves with an equal volume of Agrobacterium EHA-105 containing a pBIC binary vector with the $\mathrm{p} 19$ suppressor of Tomato Bushy Stunt virus (pBICp19). The p19 suppressor was used to ensure that the PVX sequences would not be silenced by the host post-transcriptional gene silencing mechanism.

\section{Results}

The CPEC reactions usually did not resolve as single molecular weight products in agarose gels, but rather a range of products with the approximate sizes of the expected CPEC end products were produced (Figure 2). We found that limiting the number of PCR cycles (less than 20 cycles) produced more viable colonies than did a greater number of PCR cycles (more than 20 cycles).

The E. coli (DH5 $\alpha$ ) and Agrobacterium (GV3101) transformations from two or three RT-PCR $\mathrm{CPEC}$ reaction products, referred to as reactions ' $a$ ' and ' $b$ ' (Figure1) each yielded several hundred colonies per plate when grown on LB medium. Five colonies were selected for plasmid extraction from both the DH5 $\alpha$ plates and the GV3101 plates. Plasmids were linearized with SpeI and their sizes were determined relative to the SpeI-digested PVX-pGR-106 linearized plasmid by resolving in 1.2\% agarose gels. Clones of the correct size were selected and digested with with HindIII to confirm their correct assembly (data not shown). Two clones from each of the CPEC reactions ' $a$ ' and ' $b$ ' in DH5 $\alpha$ were reserved for further molecular analyses and two clones from each of the CPEC reactions ' $a$ ' and ' $b$ ' in GV3101 Agrobacterium were used to infiltrate in $N$. benthamiana plants as described above. The plants were kept in a growth chamber at $25^{\circ} \mathrm{C}$ with 16 -hour day-length for three weeks to allow development of the mild mosaic symptoms typical of PVX infection in this host. All six plants developed symptoms in systemic leaves. PVX infections were confirmed by ELISA using PVX polyclonal antibodies (Agdia Inc, IN, USA) (Figure 3). Three plants were further confirmed to be infected by PVX using RT-PCR with PVX coat protein-specific primers (data not shown). Resulting RT-PCR products were also sequenced to confirm PVX infection with the CPEC generated PVX clone.

\section{Discussion}

Transforming the CPEC reaction products directly into Agrobacterium bypasses the need for subcloning in E. coli. An additional advantage of transforming Agrobacterium is that viral sequences that may be unstable in E. coli may be more stable in Agrobacterium, thus alleviating potential toxicity problems that have been encountered when many plant viral genomes are introduced into E. coli. In addition, this approach maintains any heterogeneity inherent in the virus population in the final full-length clones (Edmonds et al., 2013). This technique can be further modified using cell-free transformation systems, such as biolistic 


\section{Macrothink}

delivery, to completely eliminate any potential cell toxicity issues. Our approach might be capable of producing infectious clones of plant viruses that exist as quasi species such as Citrus tristeza virus (CTV) and Grapevine leaf roll associated viruses (GLRaVs) (García-Arenal et al., 2001) that have less than $10 \%$ difference between strains. CPEC-assembled full-length cDNA fragments would retain the viral heterogeneity in such cases.

Unlike other cloning methods, CPEC relies solely on the simple and robust polymerase extension mechanism to clone multiple fragments into a single construct. This technique can be routinely used to clone single genes in just five minutes depending on the complexity of the reaction (Quan \& Tian, 2009), and requires only small amounts of template DNA. The molar quantities of the RT-PCR fragments that are to be assembled in the CPEC reaction must be carefully adjusted to produce full-length infectious clones. Because the CPEC reaction is not an amplification process, the incorporation of mutations into the final product is less likely. However, excessive numbers of cycles should be avoided since this has been shown to lead to concatamer formation (Quan \& Tian, 2009). We observed that fewer cycles of CPEC reactions yielded more clones containing the correct constructs than did CPEC reactions with a larger number of cycles (data not shown). We also found that all overlapping regions should be designed to have similarly high melting temperatures (typically $55-70^{\circ} \mathrm{C}$ ) to minimize non-specific annealing.

CPEC offers a novel means to generate infectious clones of viral genomes that have limited numbers of unique restriction sites available for traditional ligation-based cloning. It also avoids the possibility that many viral genomes may be recalcitrant to cloning in $E$. coli cells because of toxicity effects. Moreover, the introduction of mutations during the various steps of conventional restriction enzyme digestion and cloning are minimized using this technique. 

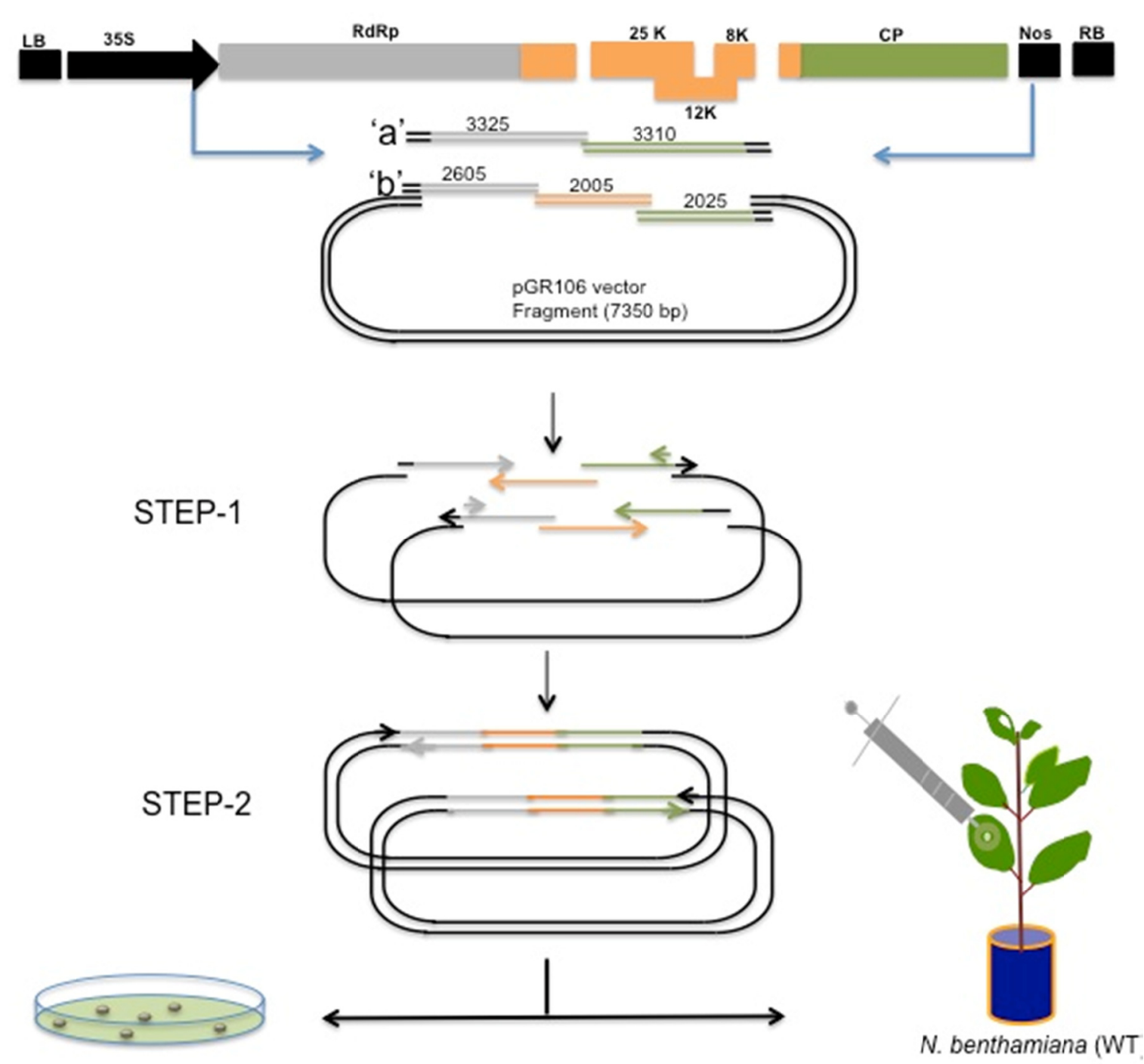

Figure 1. Generation of infectious cDNA clone of PVX by CPEC

Schematic representation of the PVX genome is shown at the top of the figure with individual virus genes, $35 \mathrm{~S}$ promoter, NOS terminator, left border (LB) and right border (RB) as indicated (figure not to scale). Overlapping colored double lines represent two or three RT-PCR fragments (size in base pairs are as indicated) of a full-length PVX clone. The two or three RT-PCR fragments together with the vector fragment (represented by black double lines) referred to as reactions " $a$ " and " $b$ " were used in the CPEC reaction to assemble a full-length PVX clone as shown in steps 1 and 2 (for simplicity, only CPEC reaction " $b$ " has been shown). In step 1, with the addition of Taq polymerase, the two or three viral PCR fragments plus the vector fragment are extended using the other as templates resulting in a complete molecule with nicks in each strand. In step 2, the nicks are sealed when the cloned molecule is introduced into Agrobacterium tumefaciens. The clones in Agrobacterïum can then either be plated on solid media or cultured in liquid media before being infiltrated into $N$. benthamiana plants. 


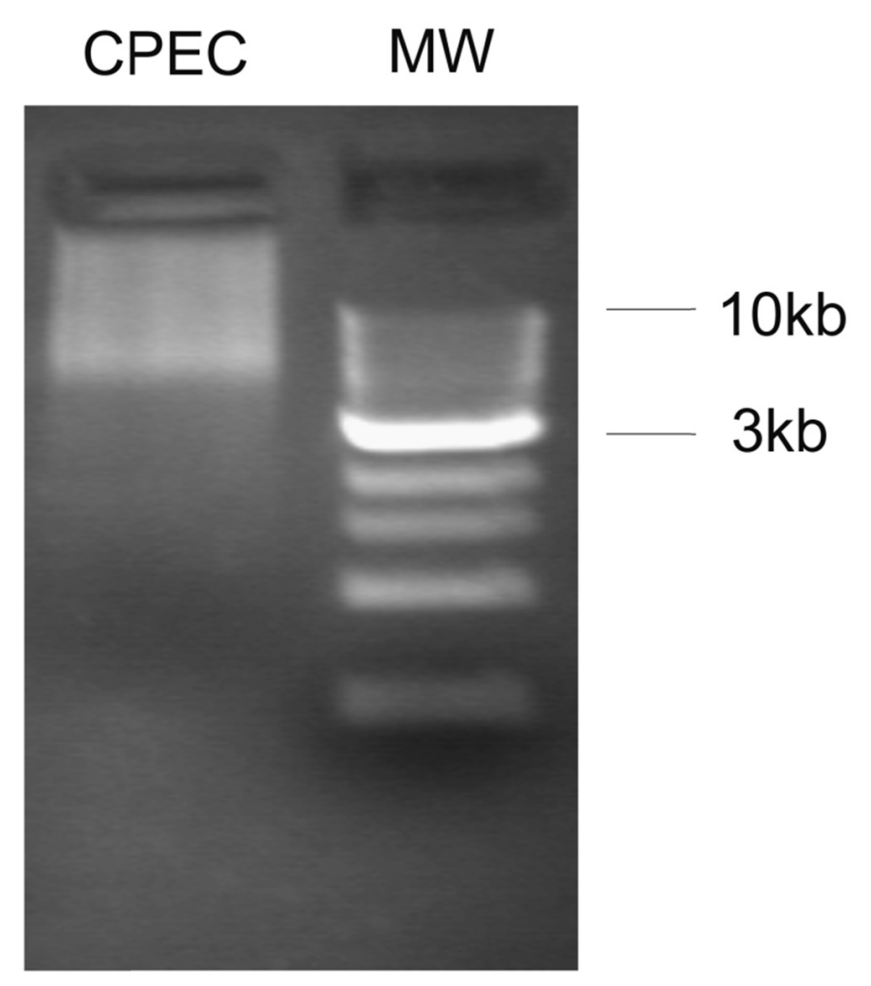

Figure 2. Gel electrophoresis analysis of the CPEC reaction

CPEC reaction products produced using three RT-PCR fragments (as noted in "b" of Figure1) generated after 15 reaction cycles. $5 \mu$ of the reaction was resolved on $1 \%$ agarose gel and visualized after ethidium bromide staining. The molecular weight marker is a $1 \mathrm{~kb}$ DNA ladder. 


\section{ELISA}

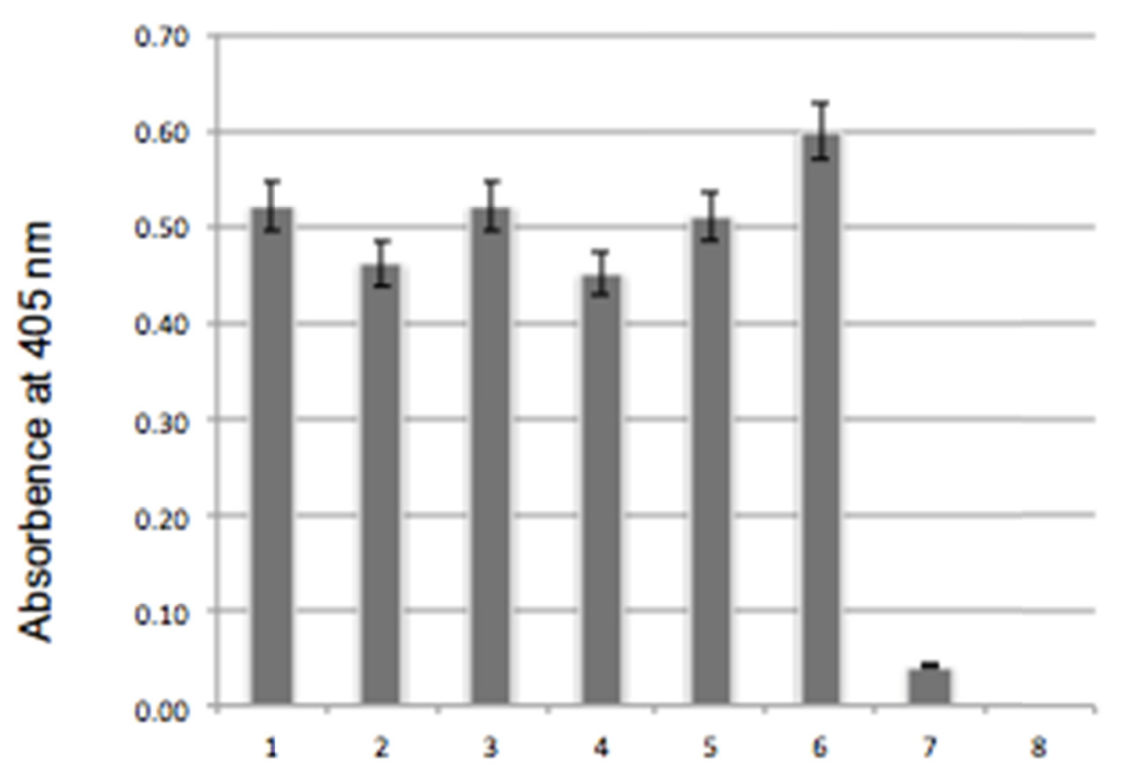

Figure 3. ELISA detection of PVX from individual systemic leaves of $N$. benthamiana agroinfiltrated with CPEC-generated PVX infectious clone (2 weeks post infiltration)

Samples 1, 2 and 3 are from plants separately inoculated with CPEC reaction 'a'; samples 4, 5 and 6 are from plants inoculated with CPEC reaction ' $b$ ' as noted in Figure 1. Sample 7 is a buffer only control. Sample 8 is from a healthy plant. All absorbance values are means $+/-$ standard error of two replicate wells per sample.

\section{Acknowledgement}

We would like to thank Dr. Judy Edmonds (University of Queensland) for helpful discussions and critical review of the manuscript and Prof. David Baulcombe for providing PVX vector. K.K.D. is a recipient of Monsanto graduate fellowship from Monsanto Co., St. Louis, MO 63166.

\section{References}

Edmonds, J., Van Grinsven, E., Prow, N., Bosco-Lauth, A., Brault, A. C., Bowen, R. A., Hall, ... Khromykh, A. A. (2013). A novel bacterium-free method for generation of flavivirus infectious DNA by circular polymerase extension reaction allows accurate recapitulation of viral heterogeneity. J. Virol, 87, 2367-2372. http://dx.doi.org/10.1128/JVI.03162-12

García-Arenal, F., Fraile, A., \& Malpica, J. M. (2001). Variability and genetic structure of plant virus populations. Annu. Rev. of Phytopathol, 39, 157-186. http://dx.doi.org/10.1146/annurev.phyto.39.1.157 
Gibson, D. G., Young, L., Chuang, R. -Y., Venter, J. C., Hutchison, C. A., \& Smith, H. O. (2009). Enzymatic assembly of DNA molecules up to several hundred kilobases. Nat. Methods, 6, 343-345. http://dx.doi.org/10.1038/nmeth.1318

Jakab, G., Droz, E., Brigneti, G., Baulcombe, D., \& Malno, P. (1997). Infectious in vivo and in vitro transcripts from a full-length cDNA clone of PVY-N605, a Swiss necrotic isolate of Potato virus Y. J. Gen. Virol, 78, 3141-3145.

Johansen, I. E. (1996). Intron insertion facilitates amplification of cloned virus cDNA in Escherichia coli while biological activity is reestablished after transcription in vivo. Proc.NatL Acad. of Sci. U.S.A, 93, 12400-12405. http://dx.doi.org/10.1073/pnas.93.22.12400

Li, M. Z., \& Elledge, S. J. (2007). Harnessing homologous recombination in vitro to generate recombinant DNA via SLIC. Nat. Methods, 4, 251-256. http://dx.doi.org/10.1038/nmeth1010

Lin, T. -Y., Dowd, K. A., Manhart, C. J., Nelson, S., Whitehead, S. S., \& Pierson, T. C. (2012). A novel approach for the rapid mutagenesis and directed evolution of the structural genes of West Nile virus. J. Virol, 86, 3501-3512. http://dx.doi.org/10.1128/JVI.06435-11

López-Moya, J. J., \& García, J. A. (2000). Construction of a stable and highly infectious intron-containing cDNA clone of plum pox potyvirus and its use to infect plants by particle bombardment. Virus Res, 68, 99-107. http://dx.doi.org/10.1016/S0168-1702(00)00161-1

Lu, R., Malcuit, I., Moffett, P., Ruiz, M. T., Peart, J., Wu, A. J., ... Baulcombe, D. C. (2003). High throughput virus - induced gene silencing implicates heat shock protein 90 in plant disease resistance. EMBO J, 22, 5690-5699. http://dx.doi.org/10.1093/emboj/cdg546

Quan, J., \&Tian, J. (2009). Circular polymerase extension cloning of complex gene libraries and pathways. PloS one, 4, e6441. http://dx.doi.org/10.1371/journal.pone.0006441

Quan, J., \& Tian, J. (2011). Circular polymerase extension cloning for high-throughput cloning of complex and combinatorial DNA libraries. Nat Protocol, 6, 242-251. http://dx.doi.org/10.1038/nprot.2010.181

Satyanarayana, T., Gowda, S., Ayllón, M. A., \& Dawson, W. O. (2003). Frameshift mutations in infectious cDNA clones of Citrus tristeza virus: a strategy to minimize the toxicity of viral sequences to Escherichia coli. J. Virol, 313, 481-491. http://dx.doi.org/10.1016/S0042-6822(03)00387-8

Satyanarayana, T., Gowda, S., Boyko, V., Albiach-Marti, M., Mawassi, M., Navas-Castillo, J., ... Lewandowski, D. (1999). An engineered closterovirus RNA replicon and analysis of heterologous terminal sequences for replication. Proc. NatL. Acad. Sci. U.S.A, 96, 7433-7438. http://dx.doi.org/10.1073/pnas.96.13.7433

Siridechadilok, B., Gomutsukhavadee, M., Sawaengpol, T., Sangiambut, S., Puttikhunt, C., Chin-inmanu, K., ... Mongkolsapaya, J.(2013). A simplified positive-sense-RNA virus construction approach that enhances analysis throughput. J.Virol, 87, 12667-12674. http://dx.doi.org/10.1128/JVI.02261-13

Sumiyoshi, H., Hoke, C. H., \& Trent, D. W. (1992). Infectious Japanese encephalitis virus RNA can be synthesized from in vitro-ligated cDNA templates. J. Virol, 66, 5425-5431. 


\section{Macrothink}

Youssef, F., Marais, A., Faure, C., Gentit, P., \& Candresse, T. (2011). Strategies to facilitate the development of uncloned or cloned infectious full-length viral cDNAs: Apple chlorotic leaf spot virus as a case study. J. Virol, $8,1-12$. http://dx.doi.org/10.1186/1743-422X-8-488

\section{Copyright Disclaimer}

Copyright reserved by the author(s).

This article is an open-access article distributed under the terms and conditions of the Creative Commons Attribution license (http://creativecommons.org/licenses/by/3.0/). 